\title{
VI.
}

\section{Zur Kenntniss der diffusen Hirnsklerose.}

(Aus dem Pathologischen Institut und dem Dr. von Hauner'schen Kinderspital zu München.)

Von 1)r. Hans Schmaus, Assistenten an pathologischen Institut $z$ n München.

(Hierzu Taf. IV.)

Unter dem Namen "diffuser Hirusklerose" fasst man alle diejenigen Veränderungen zusammen, bei denen eine Vermehrung des $Z$ wischengewebes vorzugsweise oder ausschliesslich hervortritt. Es gehören also hierber nicht blos die Fälle von Hirnhypertrophie, sondern auch gewisse Formen von Gehirnatrophie, soweit es sich um eine Schrumpfung von vorher hyperplasirtem Bindegewebe handelt.

Ferner finden sich solche diffuse Veränderungen im Anschluss an progressive Paralyse und bei der multiplen Sklerose als weitere Ausbreitung localisirter Heerde. In den meisten dieser Fälle findet sich die diffuse Sklerose als Nebenbefund neben anderen charakteristischen Veränderungen.

Neben solchen Fällen kommen aber auch andere vor, in denen eine diffuse Sklerotisirung als einzige Ursache eines bestimmten Symptomencomplexes angesehen werden muss.

Die klinischen Erscheinungen bieten in sicher gestellten Fällen gewisse gemeinsame Eigenthümlichkeiten, die sich sowohl in Fällen reiner Sklerose finden, als auch in solchen, deren Sklerose nur einen Nebenbefund darstellt.

Der nachstehend mitzutheilende Fall schliesst sich, soweit die nur ein paar Tage mögliche klinische Beobachtung eine Beurtheilung zulässt, der zweiten Gruppe an. Anatomisch stellt er ebenfalls einen Fall reiner Sklerose dar, zeigt aber Veründerungen, die auf einen höheren Grad der Erkrankung hindeuten. 


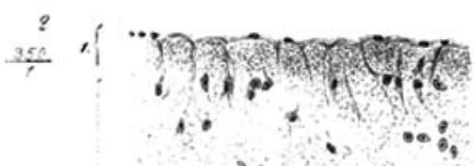

Taf: IV:
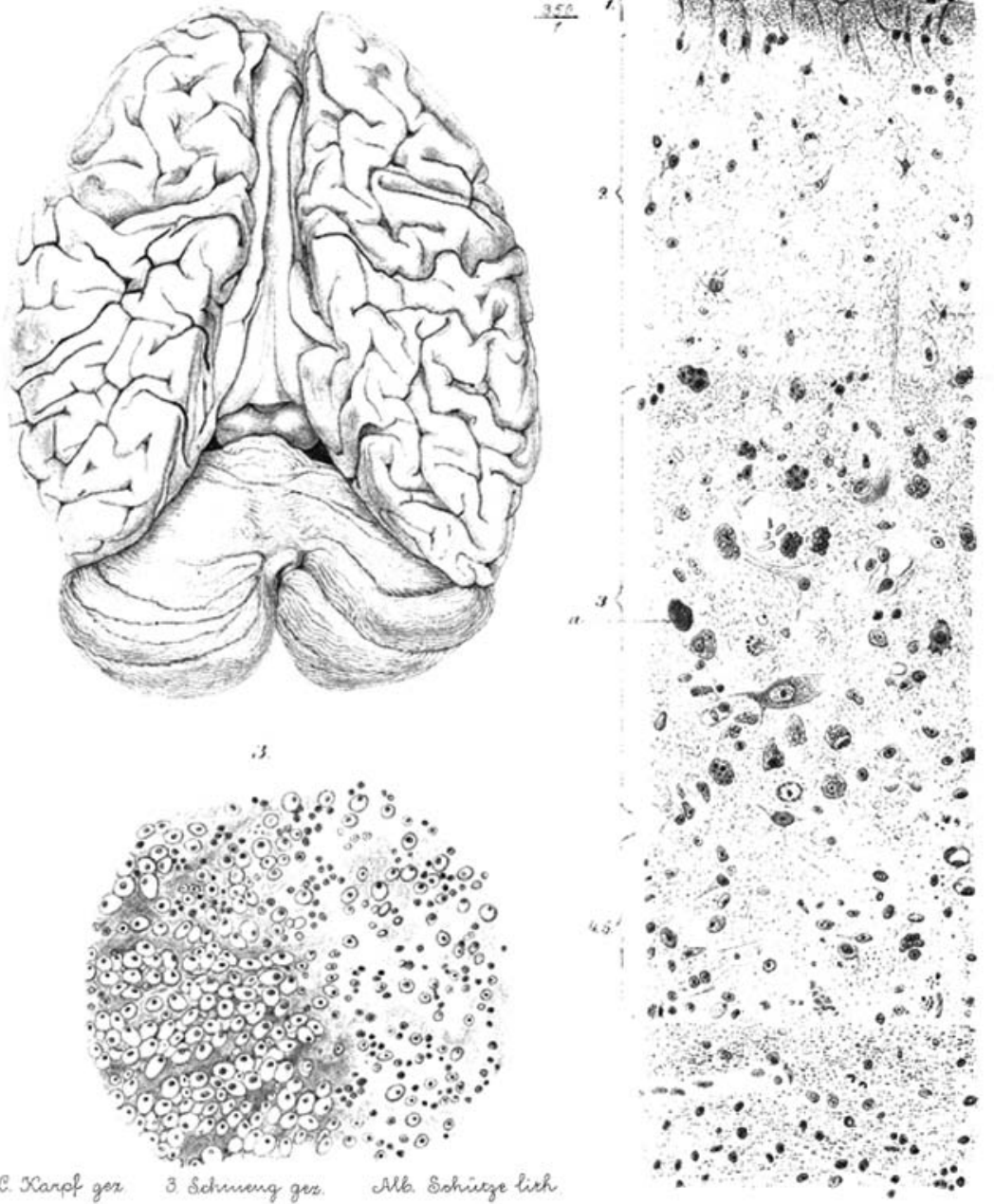
Krankengeschichte ${ }^{1}$ ).

I. Gscbwandtner, Bauerstochter aus Miesbach, geb. 21. März 1883, in das Dr. v. Hauner'sche Kinderspital aufgenommen am 7. December 1886.

Anamnese: Vater soll in der Jugend gekränkelt haben; sonst stets gesund, ebenso die Mutter. In der Familie soll keine Geisteskrankheit vorgekommen sein. Das Kind soll nach der Geburt circa $\frac{7}{4}$ Jahre gesund gewesen sein. Mit anderthalb Jahren hat das Kind reden gelernt. Im Juni 1885 erkrankte Pat. mit Kräzpfen, Fieber und psychischer Störung. Diese Erscheinungen sollen Anfangs anfallsweise aufgetreten und damals Anfschreien, Aussetzen der Respiration, Tremor vorhanden gewesen sein.

Seit einem Jahre entwickelte sich allmählich eine Contracturstellung der Extremitäten; über das Auftreten einzelner Symptome ist nichts Näheres zu erfahren. Die vegetativen Funetionen scheinen ungestört zur Zeit zu bestehen. Diarböen; Stuhl sonst norwal, angeblich schmerzhaft.

Status praesens vom 7. December 1886. Pat. für ihr Alter von entsprechender Grösse; bochgradige Blässe am ganzen Körper; Fettpolster geschwunden; Musculatur schlecht entwickelt, jedoch an den Extremitäten tetaniscbe Spannung. Pat. nimmt andauernd passive Rückenlage an, wobei der Kopf frei beweglich, dagegen die Arme in Bengecontractur mit pronirten Händen, beiderseits gegen die Brust gedrückt. Die untere Extremität in starker Extensionscontractur mit starker Varo-equinus-Stellung. Wirbelsäule beweglich. Kopf auf Druck anscheinend nicht schmerzhaft.

Augen ziemlicb tiefliegend, weit geöffuet, zeigen Strabismus divergens. Fixirung erfolgt weder am linken noch am recbten Auge. Augenbewegungen normal coordinirt; jedoch scheint die Function des linken Abducens beeinträchtigt oder aufgehoben. Pupillen weit, beiderseits gleich, auf Licht träge Reaction. Auf vorgehaltene, der Cornea genäherte Gegenstände erfolgt keine Zuckung (Amaurose?). Zeitweise ist rollende oder hin und her pendelnde Bewegung der Augäpfel zu bemerken (Nystagmus horizontalis). Gesichtsausdruck in hobem Grade schmerzhaft, obne Theilnahme nach aussen. Linke Nasolabialfalte mehr verstrichen; linker Mundwinkel weniger beweglich. Leichte Facialisparese; der linke Gaumenbogen erscbeint enger und höher als der rechte. Der Thorax ist pyrarnidenförmig gegen den Processus $x y-$ phoideus zugespitzt. Untere Lungengrenze rechterseits und Mamillarlinie an den oberen Rand der 6. Rippe verläuft horizontal. Die Auscultation ergiebt vesiculäres Athmen; an der Scapulargegend einzelne schurrende Rhonchi. Respiration etwas beschleunigt. Spitzenstoss kaum füblbar im 5. Costalraum in der Mamilarlinie; Herztöne rein, sehr frequent, Herzdämpfung nicht nachweisbar, Puls sehr beschleunigt, an der Radialis kaum füblbar, zur Zeit nicht zu zäblen. Leberdämpfung an der Mamillarlinie unter dem Rippenbogen nicbt vergrössert; Milz überragt die mittlere Axillarlinie nicht. Ab-

1) Die Krankengeschichte verdanke ich der Güte des Herrn Privatdocenten Dr. Escherich, I. Assistenten am Kiaderspital, 


\section{6}

domen kahnförmig eingesunken, Skuhl und Unin lässt das Kind unter sich gehen, jedoch bestcht kein Harnträufeln. Die oberen Extremităten sind vow Rumpe wenig abstehend, Ellenbogen unter spitzem Winkel abducirt, die Sehneu der Fingerflexoren siark vorspringend. Die Muskeln der oberen uad unteren Extremitäten, sowie die Bauchmuskeln in Contraction, stark flectirt. Finger un den Daumen fest eingeschlagen. Untere Extremitäten urbeweglich, stark extendirt. Alle Wuskeln in Spannung, Füsse in ausgesprocbener Spitzfussstellung mit Hebung des inneren Randes (equino-varus).

Mach Beugung des Kniees überzeugt man sich, dass beiderseits erhöhter Sehnenreflex vorhanden ist. Zeitweise ist ausgesprochener Dorsalclonus durch Berübrung der Achillessehne zu erzeugen; Berübrung der Fusssoble löst zu manchen Zeiten Contraction der Flexoren aus. Sensibilitat, sowie Bautreflexe scheinen normal. Muskeln der kxtremitäten zwar gering entwickelt, jedoch nicbt atrophisch. Faradische Reaction erhalten. Die Beine und Hände gewöhnlich unbeweglich gehalten, zeitweise geringe Bewegungen. Hanchwal wird der Körper wie durch elektrische Schlïge durchzuckt, was dem Kinde Schmerz zu bereiten scheint, den es durch aphonisches Wimuerm äussert. Temperatur (in ano) 38,3-38,5. Puls unter 40. Respiration 32.

10. December 18s6. Bei Verabreichung der Nahrung macht das Kind keine Schlingbewegungen. Pat. lässt Speisen aus dem Munde fallen. Auf beiden Lungen Rasselgeräusche. Am linken Unterlappen Broncbialathonen, darüber tympanitischer Seball. Sonst Status idem.

11. December 1886 Lxitus letbalis.

\section{Sectionsbefund ${ }^{1}$ ).}

Anatomisehe Diagnose: Diffuse Sklerose mit enormer Atroblie des Grosshirns besondersder Windu ugen. Hydrocephalus internus et externus ex vacuo. Pachymeningitis interna, Hydrocephalus externus. Atrophio und Vorderseiten-Strangsklerosedes Rückenmarks. - Fremdkörperpneumonie in beiden Unterlappen und im rechten Oberlappen; Abmagerung des ganzeu Körpers und seiner Organe; Beine stark gestreckt wit Varo-equinus-Stellung, Hände zur Faust geballt, zur Brust a g gezogen.

Hinterkopf vollständig abgeflacht, das hintere Drittel der Scheitelbeine bildet mit dem Hinterhaupt eine fast senkreeht abfallende Ebene.

Der Schädel giebt beim Aufsägen einen eigenthümlich boblen Klang; die Dura fest mit dem Schïdel verwachsen, zwischen ihr und den weichen Hirnbäuten in den hinteren Partien ein 1-1 th em breiter Raum, der durch belles Serum ausgefüllt ist; in den vorderen Partien ist dorselbe schmäler; weiche Hirnhäute kaum geträbt, Gefässe wenig gefüllt, Windungen im höchsten Grade atrophisch. Dieselben füblen sich - ebenso wie das ganze Gehirn sehr derb, fast lederartig an; beim Einschneiden ist die Consistenz »äh, derb.

Sectionsjournal des Pathol. Instituts No. 586. 1886. 
Ein Theil der Hirnwindungen besonders an beiden Stirn- und Scheitellappen springen gerartezu wie spitze Kämme zn, wăhend sie seitlich ron den Furchen her ungernein stark comprimirt und abgefacht sind. Zwischen den weichen Hirnhäuten ebenfalls grosse Mengen von Serum (Taf.IV. Fig. 1). Hirnkammern bedeutend erweitert.

Kleinhirn weniger atrophisch. Die Windungen daselbst unter $1 \mathrm{~mm}$; auffallendere Missbildungen, von der Atrophie abgesehen, finden sich dort nirgenis. Rüelenmark dünn, fühlt sich derb an. Dasselbe glänzt auf dem Durchschnitt ödematös, schneidet sich in seinem hinteren Umfange cirhotisch, die Höner erscheinem von grauer Farbe wie sulziges Bindegewebe. Ilin sammt Rückenmark und Dura des Rückenmarks wiegt nur $380 \mathrm{~g}^{1}$ ).

Dura an der Schädelbasis verdickt. Auf beiden Seiten der Dura firden sich Auflagerungen einer zarten Membran, in der feinste Gefüsschen stellenweise sichthar sind. An einer Stelle ein kleiner Blutpunkt. Die Entfernung des Stimbeines vom Hinterhauptbeine an der Innenseite der Schädelknochen gemessen, beträgt nur $12,7 \mathrm{~cm}$. Venensinus nicht sichtbar verschmälert ${ }^{2}$ ).

Unterlappen beider $L$ ungen sind entzündlich infiltirt, aus den Bron-. rhien ein milchig weisser Saft ausdrückbar, die grösseren Bronchien mit Eiter gefüllt. Herz normal, ebenso die Leber. Nieren hochgradig blutleer, ron blasser Farbe, Rinden- und Marksubstanz deutlich abgrenzbar. $\mathrm{Hilz}$ wenig vergrössert, Follikel geschwellt. Die Schleimhaut des aufliegenden Colon stark injicirt. Mesenterialdrüsen leicht geschwellt.

$$
\text { Histologischer Befund. }
$$

Grosshirnrinde. Färbung mit Carmin, Nigrosin, Magenta, Saffranin. Erstere machen die Ausläufer und Axencylinder besonders deutlich, letztere die Kerne und gewisse Zellformen. Die Färbung nach Weigert war an dem in Alkobol gehäteten Hirn leider nicht wehr wöglich.

In den Präparaten sind, trotzlem sie bei schwacber Vergrösserung einen ganz anderen Eindruck machen wie im normalen Hirnpräparat, die 5 Hirnschichten Meynert's ziemlich deutlich zu erkennen. Da die Begrenzung derselben sich auf die Form der Ganglienzellen stüzt, so folgt daraus einer der Hauptbefunde: Die Ganglienzellen sind jedenfalls in dei Form nicht wesentlich verändert. Wie in normalen Him finden sich hiel auch besonders grosse Ganglien, theils einzeln, theils in Gruppen, je nach den einzeluen Regionen.

1) Nimmt man nach Abzug des Rückenmarkgewichts mit beiläufig $40 \mathrm{~g}$ das Hirngewicht $\mathrm{zu} 340 \mathrm{~g}$ an, wäbrend das normale Gewicht des Gohirns eines $3 \frac{3}{4} j a ̈ h r i g e n$ Kindes mindestens $1020 \mathrm{~g}$ wiegt, so ergiebt sich, dass das Gewicht des vorliegenden Gehirns un volle $\frac{2}{3}$ geringer ist, als das eines normalen Gehirns aus diesem Alter.

2 Fine weitere Untersuchung des Gehirns in frischem Zustande bonnte wicht vorgenommen werden. 
Dagegen finden sich Abweichungen von der Norm im interstitiellen Gewebe. Zunächst sind die Ganglienzellen viel näher an einander gerückt, scheinbar zahlreicher als sonst.

Zwischen ihnen liegt nicht einfach granulirte Substanz der Neuroglia entsprechend, sondern ein feines, aber sehr dichtes Faserwerk. Dasselbe ant' seine Natur, ob Bindegewebe, ob Gliagewebe, zu prüfen, war an den gehärteten Präparaten nicht mehr möglich, indess ist es nach allen bisher gemachten Erfahrungen und der Structur wohl gerechtfertigt, dasselbe als Bindegewebe zu bezeichnen (Ziegler); wahrscheinlich, wie später sich ergeben wird, sogar ächtes Narbengewebe. Dasselbe ist an allen einzelnen Fasern fein granulirt.

Stellenweise zeigt sich eine helle Zone in der Rinde, offenbar entsprechend dem hier sehr deutlichen Baillarger'scben Streifen (die betreffenden Schnitte stammten von der dritten Stimwindung und del vorderen Centralwindung). Im Gebiete dieses Streifens ist das Gefüge der Fasern bedeutend weniger dicht; es lässt sich dieser Umstand vielleicht auf eine Verminderung der diesen Streifen bildenden Markfasem zurückführen, da das. Faserwerk in allen anderen Schichten der Rinde ziemlich gleichmässig dicht ist:

In diesem Zwischengewebe zerstreut liegen nun Zellen und Zellkörper verschiedener Art. Auffallend war ferner eine starke of hackenförmige Krümmung der von den Pyramiden ausgehenden Fortsätze, besonders der Axencylinderfortsätze. Ich glaube dieselbe auf Zerrung und theilweise Dislocation der Pyramidenzellen durch das umgebende Gewebe zurückführen zu sollen.

Im Allgemeinen spärlich und ziemlich unglejchmässig vertheilt finden sich Spinuenzellen mit Kern und einem zarten, mit seiner Substanz in das umgebende Gewebe ausstrablenden Zellleib meistens in Knotenpunkten der Fasern liegend. Dieselben färben sich im Allgemeinen schwach und sind wenig auffallend.

Dagegen sind die folgenden Formen von einem besonderen Gepräge. Ws sind meistens runde oder ovale, oft aber auch unregeluässige Körper, stark granulirt, mit deutlichem Kern, der aber ron denjenigen der Ganglien durchaus abweicht. Mit Caminfärbung fallen diese Kerne kaum auf, dagegen färben sie sich stark mit Anilinfarben.

Im Gewebe zerstreut finden sich auch Gruppen von Körnern von derselben Reaction.

Die Annahme, als seien dies degenerirte, börnig zerfallene Nervenzellen, lag nahe. Die Anilinfärbung aber deutete auf eine andere, bindegewebige Natur derselben, anf Mastzellen. Die Färbung mit Partsch-Grenacher's Carmindahlia gab die Entscheidung: Nervenzellen :oth, Kerne der fraglichen Zellen ebenfalls roth, der körnige Zellkörper blau, es sind also unzweifelhafte Mastzellen. Es muss ein lebhaftes Wachsthum derselben stattgefunden haben, denn es finden sich solche ziemlich zahlreich in Theilung und Vermehrung'). 
Neben diesen Formen, die sehr zablreich in Gewebe zarstrent waren, lagen noch Ganglienzellen vor; theils die typischen Pyramiden-, theils fortsatzlose Nervenzellen, wie in der normalen äusseren Schicht. Carmin und Nigrosin zeigten an allen die charakteristische Form, den grossen bläschenförmigen Kern mit Kernkörperchen, Axencylinderfortsat\%; der Zelleib war, leicht gekörnt, sich gut färbend; besonders Nigrosinfürbung zeigt den typischen Bau der Ganglien, namentlich den Kern gegenüber den Mastzellen sehr deutlich; Jetztere wurden mit dieser Farbe im Zellkörper blass, im Kern dunkel gefärbt, aber ohne sichtbare Kernmembran und ohne das Kernkörperchen. Zum Theil fand ich um die Nervenzellen auch die von Kreyssig beschriebenen periganglionären Räume, ein Product der Härtung ${ }^{1}$ ).

An den Gefässen fand sich nichts Besonderes; die Füllung war mässig; manchraal fanden sich Gruppen von Iyophzellen in den perivasculären Lymphscheiden; im Allgemeinen waren aber auch diese leer. In der grauen Ilirnrinde fand ich nirgends Wanderzellen.

Nach diesem allgemeinen Ueberblick folge im Weiteren eine Untersuchung der einzelnen Schichten.

Die Hirnrinde in toto ist verschmälert, ebenso die einzehnen Schichten; dieselben sind nicht mehr so gut wie normal unterscheidbar, indem die einzelnen Zellformen in einander verschoben sind; so besonders die der grossen Pyramidenschicht und die der beiden angrenzenden. Im Allgemeinen lässt sich Folgendes sagen.

1. Schicht. (Fig. 2, 1). Vorzugsweise ans Gerüstsubstanz bestehend, mit mregelmässigen kleinen Ganglienzellen.

Wie schon in der normalen Hirnrinde besteht diese Schicht vorzugsweise aus Gerüstsubstanz, die in Bündeln angeordnet ist, von denen besonders die inneren parallel zur Oberfäche verlanfen. Die Fäden des Netzwerkes sind mit feinen Körnchen besetzt. In der inneren Partie dieser Schich liegen längsgestellte Zellen von Pyramidenform. Im Gewebe zerstreut fortsatzlose Nervenkerne.

Die Pia ist verdickt und sendet in die Rinde einzelne Septa hinein.

In der ersten Schicht finden sich sehr vereinzelte Spinnenzellen, keine Mastzellen.

II. Sehicht der zahlreichen kleinen Pyramiden (Fig. 2,2). Nervenzellen zahlreich; Spinuenzellen (b) spärlich: Mastzellen (a) vereinzelt. Es überwiegen die Ganglienzellen.

III. Scbicht der grossen Pyramiden (Fig. 2, 3). Die Pyramiden

kommen von Nastzellen im Gehirn und zwar in der Umgebung von Tuberkeln.

1) Bei Färbung mit Magenta, Extraction mit Alkohol und dann noch mit Nelkenöl (nach Nissl) zeigten sich im Plasma einzelner Zellen Differenzen in der Färbbarkeit, theilweise auch stärkere Körnungen an verschiedenen Stellen des Zelllejbes, nirgends jedoch gröbere Gestaltverarrierungen desselben. 
treten etwas zurück im Verhälniss zu den hier sehr zahlreichen und grossen Mastzellen (a), sind aber immer noch zahlreicher, d. h. näher an einander gerückt, als normal. Die Mastzellen mit eingerechnet ist diese Schicht sehr reich an zelligen Elementen; auch die Spinnenzellen sind hier etwas zahlreicher.

IV. Schicht der kleinen Nervenzellen (Fig. 2, 4). Nervenzellen kaum verändert; Mastzellen noch sehr zahlreich, his an die Grenze der weissen Substanz reichend, Spinnenzellen spärlich.

In der V. Meynert'schen Schicht (Kig. 2, a) nichts Besonderes gegenüber der IV.

We isse Substanz. Lie Grundsubstanz der weissen Markmasse biltet ein Netzwerk, feiner und stäker granulirt wie die der Rinde. In demselben liegen:

1) dunkle, Anilinfarben festhaltende Kerne ohne sichtbaren Zellkörper; sie stehen dicht, regelmässig vertheilt; bei Färbung mit Methylenblan und nachfolgender Färbung mit Carmin behalten sie allein die blaue Farbe; bei der Westphal'schen Färbung auf Mastzellen bleiben sie roth. Anch halten sie Eosin fester als andere Zellen.

Jörner fanden sich in ihnen nieht. Es sind diese Formen jedenfalls den Wanderzellen beizuzählen.

2) Piazellen. In der Richtung des Faserverlaufes angeordnet, mit dentlirbem Kern.

3) In der Marksubstanz der Windungen einzelne Hastzellen.

Corpora anylacea liessen sich nicht nachweisen. Die in der weissen Substanz sebr zahlreichen Wanderzellen fehlen, wie bemerkt, in der grauen Substanz (ansserhalb der Gefässe) ganz.

Rückenmark. Die Härtung des Rückenmarks erfolgte in Müller'scher Flüssigkeit, die Färbung mit Carmin, Hämatoxylin, nach Weigert, und nach der nenerdings von $\mathrm{Pal}$ publicirten Metbode.

Das Räckenmark zeigt die Befunde einer regelmässigen combinirten Systemerkrankung, die hier als secundäre Strangdegeneration aufufassen ist. An den einzelnen Abschnitten zeigen sich die Terinderungen verschieden. Aber anch der Grad der Sklerose ist an den einzelnen Strängen verschieden. Fs finden sich Stellen, an denen sich blos eine leichte Verdickung der grösseren von der Pia her einstrahlenden Septa findet, andere mit auseinander gedrängten, theilweise zu Grunde gegangenen Nervenfasern. Aber auch nicht alle sklerotisehen Stellen können auf Strangdegeneration bezogen werden. So scharf sich die degenerirte Pyramidenbahn, die Kleinhirnbahn mit den durch anderweitige, entwickelungsgeschichtlich bekannt gewordenen normalen Bahnen der Lage nach deckt, sind andererseits auch Partien, die zwar Sklerose zeigen, aber nicht einem bestimmten Strangsystem zuzutheilen sind. Fis existirt nebenbei noch eine diffuse Degeneration.

Ich beginne die Untersuchung der einzelnen Rückenmarksabschnitte mit, den Halsmark:

Die Vorderseitenstiänge desselben sind in ihrer ganzen Ausdehnung sklerosirt, stellenweise seh" starls und scharf begrenzt, die übrigen Partien 
leicht diffus. Sehr stark sklerosirt ist die Pyramiden-Seitenstrangbabn (Fig. 3). Das interstitielle Gewebe ist stark gewuchert, die Nervenfasern auseinander gedrängt, sie sind meist zu Grunde gegangen; die Nervenscheiden durchgehends eng. - Die Axencylinder sind erhalten, manchmal, wo die Scheiden zerfallen sind, liegen sie frei in einem Hohlraum. Wo die Nervenscheiden erhalten sind, liegen die Axencylinder denselben an der Wand seitlich an. Im $Z$ wischengewebe liegen bröcklige schwarze Massen, - Ueberreste zerfallener Nervenscheiden (?). Die derartig veränderte Partie nimmt den binteren Theil der Seitenstränge ein; nach aussen ist sie durch anders veränderte Partien scharf abgegrenzt, ebenso ziemlich scharf nach vorn gegen die Substantia reticularis. Angrenzend dem grauen Hinterborn liegt sie in grösserer Ausdehnung bis zur Substantia reticularis an; es entspricht also die Lage genau der Pyramidenseitenstrangbahn.

Der Kleinhirnseitenstrangbahn entsprechend nach aussen von der Pyramidenbahn (Fig. 3) findet sich eine ebenfalls sklerosirte Partie, aber anderer Art: Von der an sie angrenzenden, stark verdickten Pia gehen dicke Septa aus, die einzelne Gruppen von Fasern abgrenzen, ohne zwisehen sie einzudringen; das feinere interstitielle Bindegewebe ist also intact. Im Gegensatz zu den vorhin beschriebenen sind die Scheiden hier erweitert. Einzelne Scheiden sind ganz geschwunden, so dass die Axencylinder frei in einem Hohlraum liegen. Die Wanddicke der Scheide ist vermindert, der Innenraum 5-6mal grösser wie in den Goll'schen Strängen. Nach vorn geht diese Partie unmerklich in die wenigen veränderten Seitenstrangreste über; sie entspricht der Kleinhirnseitenstrangbahn. Die übrigen Theile der Vorderseitenstränge zeigen keine, gewissen Bahnen entsprechende Veränderungen. Eine leichte Sklerose, bestehend in Verdickung des interstitiellen Gewebes, ist diffus äber dieselben verbreitet, die Pyramidenvorderstränge zeigen keinen höheren Grad von Veränderung, dagegen findet sich sehr hochgradige Sklerose, von der Art wie die in der Pyramidenseitenstrangbahn beiderseits am vorderen Ende des Sulcus medianus anterior und an der Austrittsstelle der vorderen Wurzeln; beide Stellen durch eine geringe sklerotische Randzone verbunden. Die Wucherung geht von der $\mathrm{Pia}$ aus und folgt zum Theil den eintretenden Wurzeln.

Ausserbalb der Pia konnte an den vorderen Wurzeln keine Sklerose gefunden werden.

Die Hinterstränge erweisen sich als vollkommen intact.

Die $\mathrm{P} \mathbf{i}$ a war nicht an allen Stellen gleichmässig verdickt, sondern am meisten da, wo sie sich an skjerotische Partien anschliesst, wenig an den Hintersträngen, stark an den Vorder- und Seitensträngen.

Besonders auffallend gestalteten sich die verschieden sklerosirten Babnen bei Färbung mit Carmin u. s. w. einerseits und der Weigert'schen Hämatoxylinmethode andererseits. In Schnitten, die in der gewöhnlichen Weise mit Carmin oder Hämatoxylin gefärbt sind, erscheint die Pyramidenbahn der Seitenstränge intensiv dunkel, in Folge der Menge des sich stark färbenden Bindegewebes. Die Kleinhirnseitenstrangbahnen dagegen sind hell,

Archiv f. pathol. Anat. Bd. CXIV. Hft. 1. 
da hier die Erweiterung der Nervenscheiden vorwiegt und die feineren Septa nicht verdickt sind. Wie die Pyramidenbahnen verhalten sich auch die anderen, als stark sklerotisch beschriebenen Partien, die wenig veränderten Vorderseitenstrangreste haben eine mittlere Nüance.

Umgekehrt ist das Verhältniss in den nach Weigert gefärbten Schnitten; in ibnen sind diejenigen Partien, welche normal geblieben sind (Binterstränge), dunkel, die anderen nm so heller, je mehr sie verändert sind: Kleinhirnbabn, Pyramidenbabn u. s. w.

Brustmark. Im Brustmark finden sich im Allgemeinen die nämlichen Veränderungen wie im Halsmark.

Die degenerirte Pyrawidenseitenstrangbahn hat eine etwas veründerte Lage. Sie grenzt nach innen an die Substantia gelatinosa und den Apex des Hinterhorns, wird aber nach innen vorn durch anderes Gewebe von der graven Substanz der Binterbörner abgedrängt. Dieses Verbalten, das genau der Lage der Pyramidenseitenstrangbahn entspricht, ist ein Beweis, dass diese hochgradig skJerosirte Partie wirklich den Pyramidenseitensträngen entspricht.

Im Uebrigen verbält sich das Brustmark wie das Halsmark.

Lendenmark. Die Kleinhirnseitenstrangbahn wird normal von der Mitte des Dorsalmarks ab durch die Pyramidenseitenstrangbahn vom Apex cornn posterioris abgedrängt, welche dadurch die Oberfläche des Rückenmarks erreicht.

In unserem Falle erstreckt sich nun auch diese Bahn entsprechend der Sklerose in derselben Art wie oben nach abwärts in das Lendenmark. Die übrigen Partien - die Kleinhirnseitenstrangbahn fehlt in diesem Theile zeigen nur leichte Veränderungen, insbesondere fehlen an den Vordersträngen die starls sklerosirten Stellen, wie sie in den beiden oberen Abschnitten beschrieben wurden. Nur einzelne vordere Wurzelfasern sind von massigerem Bindegewebe begleitet.

Graue Substanz. Die grossen Ganglienzellen der Vorderhörner sind normal erbalten, Ausläufer, Kern und Kernkörperchen deutlich; das Plasma in den Alkoholpräparaten körnig, in den wit Müller'scher Füussigkeit gehärteten finden sich die von Kreyssig beschriebenen "blassen Zellen“; die spindelförmigen Ganglienzellen der Hinterhörner der Clarke'schen Säulen und der Seitenbörner ebenfalls der Norm entsprechend. Auch die longitudinalen Bündel der Hinterhörner (Clarke's aufsteigende Colonnen von Deiters) sind ibrem Ursprung aus den binteren Wurzeln entsprechend unverändert.

Eine Uebersicht der Rückenmarkserkrankung giebt ungeführ folgendes Schema:

Die Degenerationen theilen sich zunächst in strangförmige und diffuse.

Strangförmigeden Systemerkrankungendes Rückenmarks beizuzählende Sklerosen zeigen:

1) die Pyramidenseitenstrangbahn; diese zeigt hochgradige 
Sklerose, Verdickung und Wucherung aller Zwischensubstanz mit Verdrängung und zum Theil Zerstörung der Nervenfasern;

2) die Kleinhirnseitenstrangbahn. Geringerer Grad. Wucherung der groben Septa;

Diffuse Sklerose zeigen im Allgemeinen alle übrigen Theile der Vorderseitenstränge, wenigstens im Hals- und Brustmark. Möglicher Weise ist auch diese Sklerose eine strangförmige vom Hirn absteigende; indessen lassen sich die einzelnen Bahnen nicht erkennen. Auffallend ist, dass die Pyramidenvorderstrangbahn nicht mit der Seitenstrangbahn gleichen Schritt hält;

3) die Ecken der Vorderstränge am medialen und Jateralem Ende, wo die Sklerose sichtlich von der Pia ausgeht, und eine sie verbindende schmale Randzone. Da diese letztere Sklerose mit der Piaverdickung deutlich im Zusammenhange steht, glaube ich sie als Randsklerose, selbständiger, dem Rückenmark primär zukommender Art auffassen zu müssen; um so mehr, als sich weder an den motorischen Ganglienzellen der Vorderhörner, noch an den vorderen Wurzeln ausserhalb der Pia Veränderungen nachweisen lassen.

Unter dem Namen Hirnsklerose werden sehr verschiedenartige anatomische Prozesse zusammengefasst, die nicht viel mehr als den äusseren Befund der Verhärtung, höchstens noch den der diffusen Bindegewebsentwickelung gemein haben. Nehmen wir zunächst diesen Begriff als gemeinsame Basis, so ist das Zustandekommen einer diffusen Sklerose auf zweierlei Weise zu denken.

Es kann eine localisirte "multiple" Sklerose eine grosse Ausdehnung über einen Hirnlappen erreichen und diese Fälle schliessen sich an die Heerdsklerose an. Es kann aber auch der Prozess von vornherein diffus sein, ähnlich wie die Meningitis, die progressive Paralyse. Endlich giebt es noch Erkrankungen anderer Art, die glasige Entartung der Rinde, wo die Sklerose einen diffusen, die glasige Entartung einen Heerdprozess darstellt ${ }^{1}$ ).

1) Greiff, Arch. f. Psych. XIV. 1883. Ueber diffuse und disseminirte Sklerose des Centralnervensystems und über fieckweise glasige Entartung der Hirnrinde. 
Eine Mittelstellung nehmen die diffusen Entartungen ganzer Lappen ein; deswegen und weil sie genauer gekannt sind, lässt sich vielleicht der vorliegende Fall am besten nach ihnen beurtheilen. Ich halte mich in Folgendem an die Darstellung, wie sie Wernicke in seinem Lehrbuch der Hirnkrankheiten gegeben hat.

Wernicke unterscheidet 3 Arten von Sklerose:

1) Die multiple Sklerose, ein chronisch entzündlicher Prozess mit dem Ausgang in bindegewebige Schwielenbildung.

2) Dio strangförmigen Degenerationen.

3) Umschriebene Atrophien eines Hirulappens. Hierher gehören besonders die von Cotard beschriebenen Fälle. Diese umschriebenen Atrophien sind Heilungen eines vorhergegangenen Prozesses mit Narbenbildung.

Betrachtet man nun unseren Fall qualitativ nach den gefundenen Erkrankungen -- ohne Rücksicht auf die Ausdehnung des Prozesses, so lässt er sich ziemlich sicher in dieses Schema einrechnen.

Für eine zur Zeit noch bestehende Entzündung spricht nichts; dagegen spricht das Fehlen der Wanderzellen in der grauen Sabstanz, die geringe Füllung der Gefässe, das Leersein der perivasculären Lymphscheiden. Anderseits spricht die Anwesenheit des fibrillären Bindegewebsnetzes, die Intactheit der Ganglien, für einen bereits früher abgelaufenen Prozess, der jetzt in einer relativen, anatomischen Heilung begriffen ist. Ein Beweis für die rasche Zunahme des Bindegewebes sind die zahlreichen Mastzellen, von denen man wohl annehmen darf, dass sie sich im Hirn wie in anderen Organen da finden, wo ein rasch fortschreitender Prozess von Bindegewebsneubildung vor sich geht.

Die vorliegende Sklerose wäre also das Endproduct eines interstitiellen Prozesses. Als Ursache der interstitiellen Erkrankung sieht man meistens eine Meningitis an, die im intrauterinen oder späteren Leben durchgemacht wird und auf das Hirn übergreift. Bei congenitalen Formen sind in der Regel die nervösen Elemente zerstört: auch die Krankengeschichte dentet bei unserem Fall auf eine spätere Entstehung.

Betrachten wir nun den Fall von einem anderen Gesichtspunkte, indem wir nicht von localisirten, mit der Sklerose sich 
deckenden Krankheiten, sonderu von den diffusen, wenn auch qualitativ zum Theil anderen Prozessen ausgehen.

Als diffuse hierher fallende Prozesse kann man aufstellen:

1. Die progressive Paralyse, senile Atrophie, Hypertrophie, Meningitis in ihren Ausgängen.

Von der progressiven Paralyse unterscheidet sich der vorliegende Fall principiell durch die einseitige Betheiligung des interstitiellen Gewebes. In denjenigen Fällen von Dementia paralytica, wo ein unzweifelhaft positiver Sectionsbefund vorliegt, sind die nervösen Elemente der zuerst und vorzugsweise ergriffene Theil, die Wucherung des interstitiellen Gewebes secundär. In unserer Sklerose dagegen sind die Ganglien anscheinend völlig normal, die Veränderang des interstitiellen Gewebes das primäre. Recht bezeichnend ist dabei das Verhalten der Spinnenzellen. Wo ein Untergang nervöser Elemente in grösserem Maasse stattfindet, liegen stets Spinnenzellen in grosser Zahl im Gewebe; dieselben weisen, wie Wernicke sagt, auf einen Untergang der eigentlich nervösen Gebilde hin. Bei der progressiven Paralyse sind sie nun auch sehr zahlreich, bei der Sklerose äusserst spärlich; dieses geringe Auftreten im Verein mit den zahlreich vorhandenen Mastzellen ist ein sicherer Beweis für die rein interstitielle Natur des Prozesses.

Unter dem Bilde der progressiven Paralyse verliefen manche Fälle, bei denen sich bei der Obduction eine diffuse Verhärtung des Gehirns und der Rinde heransstellte. Von den mir bekannten gehören hierher die von Zacher, Greiff und Schultze ${ }^{1}$ ) beschriebenen Fälle. Es frägt sich, ob diese Fälle von Hirnverhärtung unter dieselbe Rubrik zu setzen sind mit der sonst beschriebenen diffusen Sklerose.

Abgesehen vom abweichenden typischen klinischen Verlaufe und dem Umstande, dass neben der Sklerose zum Theil sich andere Veränderungen - glasige Entartung und andere Heerde fanden, wäre dann eine Unterscheidung zwischen dem Sectionsbefunde der Paralyse und der diffusen Sklerose überhanpt nicht

1) Zacher, Beiträge zur Pathologie und patholog. Anatomie der progr. Paralyse. Archiv für Psychiatrie. Bd. XIII. 1882. - Schultze, Ueber die Beziehungen der multiplen Sklerose des Centralnervensystems zur allgew. progressiven Paralyse der Irren, Arch. f. Psych. Bd. XI. 1880. 
mehr festzuhalten. Auch sind in dicsen Fällen die Ganglien viel mehr alterirt, die Degeneration der nervösen Elemente vorherrschend, (Spinnenzellen zahlreich!) und mehr das Bild einer zur Zeit feststehenden, floriden Entzündung vorhanden als das bei der diffusen Sklerose der Fall ist.

2. Die Meningitis kann auf das Hirn übergreifen und narbige Veränderung bewirken.

3. Hirnhypertrophie, Pseudohypertrophie, mit folgender Schrumpfung des Narbengewebes; ein ätiologisch jedenfalls ganz unklarer Begriff, ein Folgezustand verschiedener Affectionen, in manchen Fällen vielleicht mit der diffusen Sklerose identisch.

4. Senile Atrophie. Primär die Ganglien betreffend, hat daher mit unserem Falle nichts zu thun.

Im Vorstehenden wurde versucht, unseren Fall in das System der ihm ähnlichen in Betracht kommenden Hirnerkrankungen einzureihen, und den Begriff der diffusen Sklerose soweit es sich aus diesem einzelnen Falle ergiebt, ganz individuell aus demselben festzustellen.

Es folge nun ein kurzer Vergleich der unter dem Namen der diffusen Hirnsklerose bisher beschriebenen Erkrankungen, soweit sie mikroskopisch untersucht sind. Ich halte mich dabei an die Zusammenstellung von Erler ${ }^{1}$ ):

1) Strümpell (Arch. f. Psych. IX. Ueber diffuse Birnsklerose). Wucherung und Schrumpfung des Neuroglia, Erweiterung der Gefässscheiden, anscheinend normale Ganglienzellen. Chron. Encephalitis (?). Ganglien: Nicht scharf abgegrenzt; in vielen fehlt der Kern, der Inhalt trüb.

2) Kelp (Hirnskleroșe. Deutsch. Archiv f. klin. Med. X). Vermebrte Gefässentwickelung, die ganze Corticalis von einem dichten Gefässnetz durchzogen; in den Gefâsswandungen starke Kernwucherungen, jedoch nicht in allen. Grundsubstanz bomogen, darin keine Fibrillen; eingelagert in die Grundsubstanz zahìreiche Körncbenzellen, die in Gruppen beisammen liegen.

3) Erler (Diss. Tübingen 1881. Ueber diffuse Hirnsklerose). Leichte, auf ein inveterirendes Oedem zu beziehende Veränderung des Bindegewebes. Sonst normal.

4) Echeverria ${ }^{2}$ ) (citirt nach den Jabresberichten von VirchowHirscb. X. 3). Nervenelemente (in der weissen oder grauen Substanz?) vermindert, durch bindegewebige Fasern und Bindegewebszellen ersetzt.

3) Erler, Ueber diffuse Hirnsklerose. In.-Dissert. Tübingen 1881.

2) Sclerosis of both anterior frontal convolutions without aphasia. NewYork med. Record. March 1. 
0) Schüle (Deutsch. Archiv f. klin. Hed. VIll. S. 223. Weiterer Beitrag zur Hirn-Rückenmarksklerose). Grundsubstanz stark granulirt; feine Fibrillen. Ganglien degenerirt; Schrumpfung und degenerative Atrophie derselben. Schollige, glasige Entartung; diese letztere Veränderung in Heerden. Sonst diffus. Wucherung der Gefässwandungen. Metamorphose derselben zu Fibrillenzügen. Blutungen, Wucherung der Protoplasmasubstanz, "welche in den Anfängen weich, nach und nach derber und spröder wird, entweder so oder durch ibre Fahigkeit sich in Fibrillen zu spalten die uwgebenden Parenchymtheile bald mehr auseinanderdrückt, bald nur einbüllt oder verkittet".

Wie man sieht, stimmen im Allgemeinen die Befunde äberein, im Detail aber zeigen sich zahlreiche Abweichnngen. Am constantesten ist die Veränderung des interstitiellen Gewebes, die sich in den 5 Fällen 4 mal findet. Der einzige von Erler angegebene Fall mit negativem Befund scheint mir keinen directen Widerspruch zu geben; vielleicht ist die angegebene Veränderung des Bindegewebes irgend ein Stadium des sklerotischen Prozesses. Die Art der Veränderung der Zwischensubstanz wird verschieden angegeben, einmal Neuroglia- und Bindegewebswucherung, dann wieder homogene Substanz ohne Fibrillen. Die Annahme Schüle's würde eine Vermittlung herstellen, in der Weise, dass aus der homogenen Masse die Fasern sich entwickeln.

Veränderungen der Gefässwände fanden sich nicht überall. In meinem Falle, der in der Entwickelung einer interstitiellen Substanz sehr vorgeschritten war, konnte ich eine solche Veränderung nicht entdecken.

Veränderung der Ganglienzellen: In 2 Fällen wird eine Degeneration, in einem eine Verdrängung derselben angegeben.

Fasst man die Bildung der homogenen Grundsubstanz als erstes, die Fibrillenentwickelung als zweites Stadium auf, so ist die Degeneration der Ganglien der Zeit nach eine sehr ungleichmässige; 2 mal im 1. Stadium degenerirt, 2 mal (bei $S$ trü mpell und in meinem Falle) im 2. Stadium noch normal.

Jedenfalls folgt daraus, dass die Betheiligung der Nervenzellen, wenn wirklichalle diese Fälle einem Krankheitstypus angehören, eine secundäre ist; dieselben können sich jedenfalls bis in ein sehr spätes Stadium erhalten.

Zeichen der Entzündung fanden sich in 4 von den unter- 
suchten 6 Fällen; oin der Annahme einer Entzündung widersprechender Befund lag auch in den anderen Fällen nicht vor. (Erler und Echeverria.) Besonders gehört hierher die Veränderung der Gefässscheiden, Vermehrung der Gefässe, Bildung von neuem Bindegewebe, welches sich durch die Anwesenheit der Zellen als Narbengewebe definiren lässt.

Nach all' dem halte ich die Annahme für gerechtfertigt, dass die diffuse Hirnsklerose eine chronische interstitielle Entzündung darstellt, bald mit vorwiegender Betheiligung der Gefässwand, bald des interstitiellen Bindegewebes, mit Ausgang in Narbenbildung. Die meisten Fälle gehören zur Zeit der Untersuchung diesem 2. Stadium an; wenn der Prozess lange genug dauert, so treten auch an den Ganglien anatomische Veränderungen auf.

\section{Rückenmark.}

Gleichzeitige Erkrankungen des Rückenmarks sind bei diffuser Hirnsklerose mehrmals gefunden worden. Makroskopische Veränderungen fanden sich in den meisten Fällen, genaue mikroskopische Uutersuchungen wurden von Schüle gemacht. Die Art der Veränderung wird verschieden bezeichnet: Sklerose, Erweichung, Amyloiddegeneration. In allen Fällen fanden sich unregelmässige diffuse oder in Plaques vertheilte Veränderungen.

Schüle ${ }^{1}$ ) fand in seinem genau untersuchten Falle im Rückenmark denselben Prozess wie im Hirn und bezeichnet ihn als zweifellose chronische Myelitis. Eine Systemerkrankung fand er nicht, aber in der weissen Substanz zweierlei Formen, eine diffuse, an den normalen Bau des Bindegewebes sich anschliessende, and eine inselartige, die sklerotischen Plaques. Indess ist dieser Unterschied nur in der Anordnung, wicht in qualitativer Erkrankung begründet.

Der, von mir untersuchte Fall anterscheidet sich von den angeführten vor allem durch das Vorhandensein einer Systemerkrankung, neben der diffusen. Der letztere ist wahrscheinlich mit der diffusen Form Schüle's identisch; auch letztere schliesst

1) Scbüle, Weiterer Beitrag zur Hirn-Rückenmarksklerose. Dentsches Archiv f. klin. Medicin. VIII. S. 223. 
sich deutlich dem normalen Bau dos bindegewobigen Gerüstes an. Verbreiterung der Septa, welche die Axencylinder auseinanderdrängen, secundäre Veränderung der nervösen Elemente. In dem Falle Schüle's ist das die einzige Form die zur diffusen Sklerose des Gehirns gehört, denn auch im Hirn war neben der diffusen noch eine multiple Erkrankung vorhanden, dem analog im Rückenmark. Strangförmige, combinirte Systemerkrankungen fand ich in der Literatur der diffusen Sklerose nicht verzeichnet. Sicher gehört hierher die Degeneration der Pyramidenseitenstränge, vielleicht auch der Kleinhirnseitenstränge und der Vorderstränge; zur diffusen primären Form gehört die Randsklerose.

Das Rückenmark ist also primär weniger betheiligt wie in dem Falle von Schüle.

Die Hauptveränderung ist rein secundär, was sich auch aus der ausschliesslichen Erkrankung der weissen Substanz ergiebt. Die motorischen Ganglien waren hier intact, die secundäre Degeneration erstreckte sich also auf den ersten Abschnitt der motorischen Bahn, die Seitenstränge bis zu den Ganglienzellen der Vorderhörner.

Die diffuse Hirnsklerose verbält sich also in dieser Beziehung nicht anders wie die multiple, und zahlreiche andere Hirnerkrankungen.

Symptome. Wenn auch ein geschlossener Symptomencomplex der diffusen Sklerose bis jetzt nicht existirt, so ist doch den meisten Fällen eine Reihe bestimmter Erscheinungen gemeinsam. Dass dieselben nicht überall als die einzigen and constant vorkommen, ist um so leichter erklärlich, als unter dem Namen der Krabkheit nach dem Obigen vielleicht sehr verschiedene Prozesse aufgeführt werden. Andererseits können ja auch verschiedene Erkrankungen in ihren Wirkungen sich vollkommen gleichen, besonders wenn es sich wie hier vorzugsweise um diffuse nicht localisirte Prozesse handelt.

Im Allgemeinen wiegen auch die diffusen Symptome über die Heerdsymptome vor, und zwar sind es vorzugsweise die der raumbeschränkenden Erkrankungen und besonders Erscheinungen von Reizung der Hirnrinde. Dieselbe äusserte sich durch epileptiforme Anfälle, den Tremor, die Convulsionen im Beginne der Krankheit, Auch fanden sich in diesem Falle die von 
Strümpell, Schüle, Kuessner') gefundenen rhythmischen Zuckungen. Auch in dem Falle von Kelp, der neben der von Strümpell beschriebenen Sklerose dem vorliegenden wohl am nächsten steht, fanden sich neben den Convulsionen in den anfallsfreien Zeiten fortwährend oscillirende Zuckungen.

Der Tremor war ein continuirlicher, kein Intentionstremor. In einem von Greiff beschriebenen Falle, der allerdings auch diffuse Sklerose zeigte (im weiteren Sinne), war noch eine multiple Erkrankung mit combinirt.

Diese Symptome erklären sich alle aus der Degeneration der Rinde, ebenso die psychische Störung.

Ferner finden sich andere Symptome, die von einer Läsion der Pyramidenseitenstrangbahn herrühren; da diese in ihrem ganzen Verlauf lädirt ist, so kann die Motilitätsstörung nichts Auffallendes haben; dem Befunde entsprechen auch die spastischen Symptome; es handelt sich um eine centrale Lähmung.

Einfache Atrophie, keine Entartungsreaction, erböhte Reflexe stimmen ebenfalls mit diesem Befunde. Die Reflexerhöhung rührt möglicherweise von der Zerstörung der reflexhemmenden Fasern in den Seitensträngen her; indess muss man sich in diesen Fällen, worauf Schultze aufmerksam macht, hïten, dieselbe ohne Weiteres als spinale Erscheinungen aufzufassen, denn gerade in Fällen obne Rückenmarkserkrankung wurden diese Erscheinungen auch gefunden bei völlig intactem Rücken$\left.\operatorname{mark}^{2}\right)$.

Jedenfalls aber war die Ursache der Lähmang oberhalb der grauen Vorderhörner und daher die spastische Lähmung durch die Leichendiagnose gerechtfertigt.

Die geringe Betheiligung des sensiblen Systems ist auch anderen Fällen gemeinsam; jedenfalls erstreckte sich eine etwaige Degeneration sensibler Elemente nicht auf das Rückenmark.

Von anderen Heerdsymptomen sind zu nennen die motorische, träge Pupillarreaction, Nystagmus.

Bulbäre Symptome sind durch Sklerose in der Oblongata erklärt. Indess lässt sich wohl bei einem Prozess, der das Hirn

1) Arch. f. Psych. Bd. VIII. Athetose-Bewegungen bei einem Paralytiker ohne Heerderkrankung im Gehirn.

9) Zacher, Arch. f. Psych. XIII, 1882. 


\section{1}

wie die gesammte motorische Bahn so allgemein ergreift, der Sitz der Ursache nicht bestimmen.

Der Sectionsbefund erklärt also vollständig alle klinischen Erscheinungen. Nur eine Veränderung wenigstens der motorischen Ganglien, die man a priori hätte voraussetzen können, fand sich nicht. Wenn man - was nach neueren Forschungen zweifelhaft geworden ist - den Ursprung der Innervation in den Ganglien der Hirnrinde annimmt, so erklären die interstitiellen Prozesse doch eine Reizung der Nervenzellen, abgesehen davon, dass dieselben Veränderungen erleiden können, die für unsere Hülfsmittel nicht nachweisbar sind.

Nimmt man aber den neueren Ansichten zu Folge die Fasern der Hirnrinde selbst als die nervösen Endorgane an, so spricht die gesetzmässige, symmetrische Erkrankung der Pyramidenbahnen, die von einem Theil derselben die unmittelbare Fortsetzung sind, für ihre Erkrankung. Bemerkt sei noch, dass die Axencylinderfortsätze der Rindepyramiden stets deutlich erhalten waren.

Es folgt eine Zusammenstellung der wichtigsten in der Literatur angegebenen Symptome (nach Erler), wobei das Zutreffen in meinem Falle mit +, das Fehlen mit - bezeichnet ist.

Paresen, Paralysen der Extremitäten 7 .

Facialisparese 7

Incontinentia alvi 6

$$
\text { urinae } 6
$$

Augenmuskellähmung 2

Pupillenveränderung 2

Sprachstörung 9 (?)

Schlingbeschwerden 5

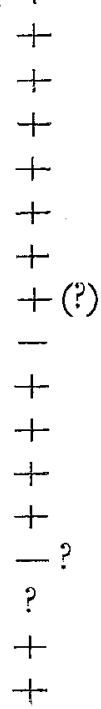

Apoplectiforme Anfälle 5

Epileptiforme Anfälle 4 (3?)

Contracturen 3

Zuckungen 9

Tremor 4

Sensibilitätstörung - ?

Kopfschmerz 2

Psychische Störungen 7

Erhöhung der Reflexe 
Zum Schlusse erlaube ich mir Herrn Prof. H. Rauke, Director des von Hauner'schen Kinderspitals, für die gütige Ueberlassung des Falles, Herrn Prof. Bollinger für die eingehende und vielseitige Unterstützung bei der Bearbeitung meinen besten Dank auszusprechen.

\section{Nachtrag.}

Neuerdings erschien in der "Berliner klinischen Wochenschrift" 1887 No. 49 eine Mittheilung von Dr. Buss "Ueber einen Fall von diffuser Hirnsklerose", aber erst nach Abschluss der vorliegenden Arbeit und konnte daher nicht mehr berücksichtigt werden.

\section{Erklärung der Abbildungen. \\ Tafel IV.}

Fig. 1. Gebirn von oben, in Alkohol eingelegt. Windungen schmal, kammförmig, scharfe kantige Formen. Das Kleinbirn ist fast normal gross, das Grosshirn bat nur $\frac{1}{3}$ des gebörigen Volumens. Die Windungen sind zablreich, unregelwăssig.

Fig. 2. Färbung mit Magenta. $350: 1$. Schnitt durch die Hirnrinde. Die 5 Schichten nach Meynert mit den beschriebenen Veränderungen. I Schicht der vorwiegenden Gerüstsubstanz mit kleinen Nervenzellen. 2 Scbicht der kleinen Pyramiden. a Nastzellen. b Spinnenzellen. 3 Schicht der grossen Pyramiden. 4, 5 Sehicht der inneren Pyramiden; nach innen davon die Marksubstanz der Gyri.

Fig. 3. Querschnitt des Halsuarks. Färbung nach Weigert. 350:1. Rechts zeigt sich die stark sklerosirte Pyramidenbahn, in der Mitte die äussere Grenze, links die Kleinhirnseitenstrangbahn, die von einzelnen verdickten Septen durchsefzt ist; die Nervenscheiden sind blau, das Bindegewebe gelb. Das Gesichtsfeld ist der Grenze der Pyramidenseitenstrang- und der Kleinhiruseitenstrangbahn des linken Seitenstranges entnommen. 